\title{
Correction to: Environmental temperature and relative humidity differently affect the sperm characteristics in Brown Swiss and Belgian Blue bulls
}

\author{
Alessia Gloria $^{1} \cdot$ Luca Candeloro $^{2} \cdot$ Laura Wegher $^{3} \cdot$ Domenico Robbe $^{1} \cdot$ Augusto Carluccio $^{1} \cdot$ Alberto Contri $^{4}$ \\ Published online: 7 October 2021 \\ (c) ISB 2021
}

Correction to: International Journal of Biometeorology https://doi.org/10.1007/s00484-021-02184-z

The article was originally published with incorrect author name sequencing. This correction stands to correct the original article. The original article has been corrected.

The original article can be found online at https://doi.org/10.1007/ s00484-021-02184-z.

Alessia Gloria agloria@unite.it

1 Faculty of Veterinary Medicine, University of Teramo, Loc. Piano D'Accio, 64100 Teramo, Italy

2 Istituto Zooprofilattico Sperimentale Dell'Abruzzo E del Molise, Campo Boario, 64100 Teramo, Italy

3 Provincial Breeders Federation of Trento, Via delle Bettine 40, 38121 Trento, Italy

4 Faculty of Biosciences and Technologies for Agriculture, Food and Environment, via Balzarini 1, 64100 Teramo, Italy 